\title{
Research on Multi-Cooperative Combine-Integrated Scheduling Based on Improved NSGA-II Algorithm
}

\author{
Li Ma, College of Engineering, Northeast Agricultural University, Harbin, China \\ Yidi Wang, College of Engineering, Northeast Agricultural University, Harbin, China \\ Meiqiong Ma, JI Baiwang Technology Co., Ltd., Shenzhen, China \\ Jiyun Bai, College of Arts and Sciences, Northeast Agricultural University, Harbin, China
}

\begin{abstract}
To promote the integration and optimal allocation of agricultural machinery resources to achieve the purpose of reducing cost and increasing efficiency, the scheduling problem of agricultural machinery in agricultural machinery cooperatives based on the trans-regional operation mode was studied in this paper, Considering multiple agricultural machinery points, multiple types, operation time windows, space distance, and other factors, the multi-objective programming mathematical model with the lowest total cost of deployment, the highest service punctuality, and the least use of harvester was established by applying path optimization and theory of job shop scheduling. NSGA-II was used to solve the model in this paper. According to the model features, this paper designed chromosome coding and the process of emergence, crossover, and variation of initial population. Combined with the actual situation of rice harvesting in Wuchang City, the above scheduling theory was applied. The experimental results showed the validity and feasibility of the scheduling model and the algorithm.
\end{abstract}

\section{KEYWORDS}

Combine, Multi-Objective Optimization, NSGA-II, Scheduling Model, Trans-Regional Operation

\section{INTRODUCTION}

With the expansion of the agricultural machinery operation scope and the increase of the number of harvesters, there were fierce competition in the trans-regional operation market of combine in recent years. And the scheduling problem of combine was growing one of the main factors affecting the economic benefits of trans-region operation (Liao et al., 2008). Due to the lack of meticulous and systematic planning in current trans-regional operations. there were still many problems, such as unreasonable sequence of operations on plots, low work efficiency, and high operating costs; unreasonable resource allocation for harvesters, resulting in waste of resources;Coupled with roads and weather and other factors, the harvester cannot arrive on time, thus delaying the farming time.

The essence of trans-regional scheduling of combine was a resource scheduling problem between agricultural machinery and farmland with time window and space distance constraint.In the past ten years, many scholars have applied a variety of planning methods and intelligent optimization algorithm to carry out a large number of research, and achieved many results (Foulds et al.,2005,\& Zhang,2006,\& Dionysis Bochtis et al.,2007,\& Ferrer et al.,2008,\& Li et al.,2008,\& Guan et al.,2009,\& 
Wang et al.,2010).Zhang (2012) studied the of agricultural machinery scheduling based on owner selection. Wu (2013) established a time-window constrained scheduling model for agricultural machinery and solved the it with dynamic programming. Wang (2013) studied the scheduling of cotton pickers as VRP-TW problem. Giovanni (2018) solved the problems of long round-trip time and long waiting time of agricultural machinery material filling based on dynamic programming accurate algorithm and branch and bound algorithm. Hasan (2018) obtained a reasonable agricultural machinery operation path planning and scheduling method through improved Clarke Wright algorithm and tabu search algorithm. Wang (2019) established the agricultural machinery scheduling model with time window, and designed an agricultural machinery scheduling method based on genetic algorithm, and verified the model and algorithm through the test data set and the actual case of agricultural machinery scheduling. Zhao (2019) constructed the objective function according to the multi scheduling objectives of agricultural machinery steering gear workshop, and improved the immune clonal algorithm by using multi strategy population initialization and adaptive mutation operator. Wang (2020) simulated and iterated the agricultural machinery and farmland in a region by genetic algorithm, and screened out the best scheme of agricultural machinery scheduling. Wang (2020) combined heuristic rules, genetic algorithm and simulated annealing algorithm to design a three-level hybrid heuristic algorithm solving model for joint optimization of resource allocation and job scheduling of railway container central station.

It can be found that most of the current researches on combine scheduling transformed it into a VRP-TW problem or a transportation problem in operational research through the literature analysis, the scheduling was considered as only one agricultural machinery point and one type agricultural machinery, most of the modeling aimed at the lowest cost that ignored the indicators such as punctuality of service for farmland and utilization rate of harvesters. Based on the above research, this paper discussed the resource allocation of harvesters with multiple agricultural machinery points, multiple types, operation time window, space distance constraints in trans-regional operations, constructed a practical mathematical model of multi-objective programming, and used the Elitist Nondominated Sorting Genetic Algorithm (NSGA-II) to solve the problem. According to the obtained optimal scheme, combine could be effectively scheduled and reasonably distributed, and the purpose of maximizing the benefits of the machine owner, the farmer, and the trans-regional organization was finally achieved.

\section{PROBLEM DESCRIPTION AND ANALYSIS}

\section{Problem Description}

The problem of trans-regional job scheduling of multi-cooperative agricultural machinery can be described as: multiple agricultural machinery points distributed at different locations have multiple types of harvesters and need to provide harvesting service for multiple operating points. Each operation point has an optimal harvest period (time window), that is, the earliest start time and the planned completion time allowed by the point, which requires the harvester to start harvesting in the time window and finish the task as quickly as possible. The dispatcher needs to assign the appropriate type of machine, the right number of harvesters, and the appropriate working routes for each operating point so that the harvester can complete all tasks on time with the lowest cost, quality and quantity. The problems are specified as follows:

(1) The crop matures and reaches the harvest condition in a certain time, the farmer proposes the earliest start operation time and the latest planned completion time, the combine must perform the harvesting service within the time window, if it arrives at the operation point early, it must wait until the time allowed to start (Pilla et al., 2012). Due to some uncontrollable factors in the operation process, appropriate delays are allowed for the completion of farmland tasks. 
(2) The tasks of each operation point can be divided, different farmland operation points may be served by the harvester combination of different agricultural machinery points (Sheridan et al., 2013).

(3) The starting time of each harvester at the same operation point may be different, but the left time is the same. Because farmers want their own harvesting to be completed as soon as possible in the actual farm work, so once the harvesters start working, they cannot leave until it finishes the task of the operation point (Tan et al., 2016).

(4) Due to the different harvesting methods and terrain and parcel characteristics of each operation point, the structural performance requirements of the harvester are also different, so the suitability of the model and operation point must be considered in the scheduling.

(5) As the size of the operation points and the number of grain delivery machines are limited, each operation point limits the maximum quantity of harvester for simultaneous operations.

\section{Determination of Model Objectives}

According to the analysis, the trans-regional job scheduling problem of combine is a complex combinatorial optimization problem, which mainly includes three typical combinatorial optimization sub-problems: Assigning Problem (Zhao, 2013), Routing Problem (Tian, 2007) and Scheduling Problem (Yang et al., 2013). At the same time, the trans-regional scheduling of combine is also a multi-objective optimization problem. The ultimate goal is to make efficient scheduling and rational distribution of harvesters based on resource integration and utilization. This paper mainly considers the scheduling objectives from the following aspects:

(1) The lowest total cost of deployment .Trans-regional job scheduling costs mainly include the harvester transfer cost and usage cost. The transfer cost of the harvester is related to the total deployment path, the shorter the deployment path, the lower the transfer cost; The cost of using the harvester mainly refers to the fixed cost of the harvester, such as operator's salary, the management fees, the maintenance fees, the depreciation fees and so on. The same type of harvester costs the same. At the same time, the waiting cost of the harvester arriving at the operation point in advance should be considered.

(2) The highest punctuality of operation. Due to the limitation of the operation time window, the harvester should arrive at the operation point on time, finish the farmland task as quickly as possible, shorten the delay time of the end of the task at each operation point, and ensure that all tasks can be completed on time as far as possible.

(3) Minimum total number of harvester deployment. When the harvester resources are sufficient to meet the service demand for all farmland operation points, it is necessary to consider using a smaller number of harvesters to complete farmland tasks. Because, the less the number of harvesters used, the lower the cost, the higher its utilization rate.

\section{MODEL AND METHODS}

Model Assumption Conditions and Description of Variables

1. Model Assumption Conditions

a. The location of each agricultural machinery point, the type of harvesters owned, quantity, and the operational capacity of harvesters are known;

b. Location, area, time window, available model, minimum demand and maximum limit of each operation point are known;

c. The transfer cost of the harvester between different operating points is only related to the transfer distance; 
d. Each harvester starts from a farm machinery point, finishes the task and finally returns to the starting farm machinery point;

e. Ignoring the factors such as operator's driving technology, service life of the harvester and so on, the same type of harvester has the same operating capacity.

f. To facilitate maintenance and management, only one type of harvester can be assigned to each operation point.

2. Model Variables Description

Table 1. Model variables description

\begin{tabular}{|c|c|}
\hline \multicolumn{2}{|c|}{ (1) Number description } \\
\hline Number & Number description \\
\hline$V^{k}$ & Agricultural machinery point set, $V^{k}=\{1,2, \ldots, K\}$ \\
\hline$V^{i}$ & Harvester model set, $V^{i}=\{1,2, \ldots, I\}$ \\
\hline$V^{j}$ & Farmland operation point set, $V^{j}=\{1,2, \ldots, n\}$ \\
\hline$V$ & Path nodes set, $V=V^{k} \cup V^{j}$ \\
\hline$h$ & Path nodes number, $h \in V$ \\
\hline$k$ & Agricultural machinery points number, $k \in V^{k}$; \\
\hline$j$ & Farmland operation points number, $j \in V^{j}$ \\
\hline$i$ & Harvester models number, $i \in V^{i}$ \\
\hline$V^{k i}$ & $\begin{array}{l}\text { The set of model } i \text { harvesters which locates agricultural machinery points } k, V^{k i} \text { can be empty set, } \\
V^{k i}=\left\{V_{1}^{k i} V_{2}^{k i} V_{3}^{k i}, \ldots, V_{N_{k}^{i}}^{k i}\right\}\end{array}$ \\
\hline$V_{l}^{k i}$ & $\begin{array}{l}\text { Harvesters number, which represents the } l \text { th harvester of model } i \text { at the point of agricultural } \\
\text { machinery point } k \text {; }\end{array}$ \\
\hline$N_{j}$ & Set of available models for operation points $j, N_{j} \subseteq V^{j}$ \\
\hline \multicolumn{2}{|c|}{ (2) Parameter description } \\
\hline Parameter & Parameter description \\
\hline$K$ & Number of agricultural machinery points \\
\hline$I$ & Number of harvester models \\
\hline$n$ & Total number of agricultural operation points \\
\hline$N_{k}^{i}$ & Number of harvesters of model $i$ at the point of agricultural machinery point $k$ \\
\hline
\end{tabular}


Table 1. Continued

\begin{tabular}{|c|c|}
\hline$N_{i}$ & Total number of model $N_{i}$ harvesters, $N_{i}=\sum_{k=1}^{K} N_{k}^{i}$ \\
\hline$S_{j}$ & Area of work to be completed at the operation point $j$; \\
\hline$Q_{i}$ & Productivity of model $i$ harvesters, i.e. the harvesting capacity per unit time \\
\hline$\left[B_{j}, E_{j}\right]$ & $\begin{array}{l}\text { Time window for the operation point } j \text {, i.e. the earliest start operation time and scheduled } \\
\text { completion time allowed by point } j ;\end{array}$ \\
\hline$T_{j}$ & Planned duration of harvest task at the operation point $j, T_{j}=E_{j}-B_{j}$; \\
\hline$R_{i j}$ & Minimum number of harvesters required for point $j$ tasks to be performed by model $i$ harvesters; \\
\hline$Y_{i j}$ & Maximum number of harvesters required for point $j$ tasks to be performed by model $i$ harvesters; \\
\hline$D_{(h, j)}$ & Deployment distance from point $h$ to operation point $j$ \\
\hline$V_{(h, j)}$ & The speed of the harvesters from point $h$ to operation point $j$; \\
\hline$T_{(h, j)}$ & The transfer time of harvesters from point $h_{\text {to operation point }} j, T_{\leftrightarrow h, j)}=\frac{D_{(h, j)}}{V_{(h, j)}}$ \\
\hline$C_{i}$ & Operating cost of model $i$ harvesters \\
\hline$C_{(h, j)}$ & Transfer cost of harvesters per unit distance \\
\hline$C$ & Waiting cost of each harvester's per unit time. \\
\hline \multicolumn{2}{|c|}{ (3) Variable Description } \\
\hline Variable & Variable Description \\
\hline$X_{i j}$ & Whether to assign type ${ }^{i}$ harvesters to operation point ${ }^{j}$, represented by the $0-1$ variable \\
\hline$N_{i(h, j)}$ & The number of model $i$ harvesters from $h_{\text {point to operation point }} j$ \\
\hline$R_{j}$ & Whether the task completion time of operation point $j$ is delayed \\
\hline$W_{j}$ & Delay time for task completion of operation point $j$ \\
\hline$A_{(h, j)}$ & The time the harvesters from point $h_{\text {to point }} j$ \\
\hline
\end{tabular}




\begin{tabular}{|l|l|}
\hline$Z_{(h, j)}$ & $\begin{array}{l}\text { Whether the time of the harvesters arriving at point } j_{\text {from point }} h_{\text {is earlier than the earliest start }} \\
\text { time allowed by point } j\end{array}$ \\
\hline$\left[b_{j}, e_{j}\right]$ & Actual start and finish time of operation point $j$ \\
\hline
\end{tabular}

\section{Model Establishment}

\section{Objective Function}

Optimization goal 1: the total cost of deployment is the lowest, as shown in formula (1). Among them, formula (2) means minimizing the cost of harvesters' transfer and deployment, formula (3) means minimizing the cost of harvesters' use, and formula (4) means minimizing the cost of waiting in advance.

$\operatorname{Min} F_{1}(x)=f_{1}(x)+f_{2}(x)+f_{3}(x)$

$f_{1}(x)=\sum_{j=1}^{n+K} \sum_{h=1}^{n+K} \sum_{i=1}^{I}\left(x_{i j} C_{(h, j)} N_{i(h, j)} D_{(h, j)}\right)$

$f_{2}(x)=\sum_{k=1}^{K} \sum_{j=1}^{n} \sum_{i=1}^{I} x_{i j} C_{i} N_{i(k, j)}$

$f_{3}(x)=\sum_{j=1}^{n+K} \sum_{h=1}^{n+K} \sum_{i=1}^{I} x_{i j} N_{i(h, j)} Z_{(h, j)}\left(B_{j}-A_{(h, j)}\right)$

Optimization goal 2: service punctuality is the highest, which is mainly considered from two aspects: minimizing the delay time of all operation completions, as shown in formula (5), maximizing the number of operating points that complete the task on time, as shown in formula (6).

$\operatorname{Min} F_{2}(x)=\sum_{j=1}^{n} W_{j}$

$\operatorname{Max} F_{3}(x)=\sum_{j=1}^{n} R_{j}$ 
Optimization goal 3: the minimum number of harvesters used, as shown in formula (7).

$\operatorname{Min} F_{4}(x)=\sum_{k=1}^{K} \sum_{j=1}^{n} \sum_{i=1}^{I} x_{i j} N_{i(k, j)}$

The overall optimization objective function is shown in formula (8)

$$
F=\phi\left(\begin{array}{l}
\min \left\{F_{1}(x)\right\}, \min \left\{F_{2}(x)\right\}, \\
\max \left\{F_{3}(x)\right\}, \min \left\{F_{4}(x)\right\}
\end{array}\right)
$$

\section{Constraint Condition}

$$
\begin{aligned}
& Q_{i} \sum_{h=1}^{n+K} \sum_{i=1}^{I} x_{i j} N_{(h, j)}\left(e_{j}-A_{(h, j)}\right)\left(1-Z_{(h, j)}\right)+ \\
& Q_{i} \sum_{h=1}^{n+K} \sum_{i=1}^{I} x_{i j} N_{(h, j)}\left(e_{j}-B_{j}\right) Z_{(h, j)}=S_{j}, i \in N_{j} \\
& \sum_{i=1}^{I} x_{i j}=1, j=1,2 \cdots n, i \in N_{j}, j=1,2, \cdots, n \\
& \sum_{j=1}^{n} N_{i(k, j)} \leq N_{k}^{i}, k \in V^{k}, i \in V^{i} \\
& B_{j} \leq b_{j} \leq E_{j}, j \in V^{j} \\
& \sum_{i=1}^{I} \sum_{j=1}^{n} N_{i(k, j)}=\sum_{i=1}^{I} \sum_{j=1}^{n} N_{i(j, k)}, k \in V^{k} \\
& R_{i j} \leq \sum_{h=1}^{K+n} \sum_{i=1}^{I} x_{i j} N_{i(h, j)} \leq Y_{i j} \leq N_{i}, i \in N_{j}, j \in V^{j}
\end{aligned}
$$




$$
W_{j}=\left\{\begin{array}{l}
0, \quad e_{j} \leq E_{j} \\
e_{j}-E_{j}, e_{j}>E_{j}
\end{array}\right.
$$

$R_{j}= \begin{cases}0, & e_{j}>E_{j} \\ 1, & e_{j} \leq E_{j}\end{cases}$

$e_{h}+T_{(h, j)}=A_{(h, j)}, h \in V, j \in V^{j}$

$$
Z_{(h, j)}= \begin{cases}1, & B_{j}-A_{(h, j)}>0 \\ 0, & B_{j}-A_{(h, j)} \leq 0\end{cases}
$$

$R_{i j} Q_{i} T_{j} \geq S_{j}, i \in N_{j}, j \in V_{j}$

$x_{i j}=\left\{\begin{array}{l}1, \text { assign model } i \text { harvester to serve at the operation } \\ \text { point } j \\ 0, \text { do not assign model } i \text { harvester to serve at the } \\ \text { operation point } j\end{array}\right.$

$N_{i(h, j)} \geq 0$ and is an integer, $i \in V^{i}, h \in V, j \in V^{j}$

Formula (9) limits that the tasks of each operation point to be completed; Formula (10) limits each operation point to selecting only one type of harvester to serve it; Formula (11) limits the number of harvesters dispatched by each agricultural machinery points cannot exceed its maximum limit; Formula (12) limits that the actual start harvest time of each operation point must be within the time window; Formula (13) limits the number of harvesters serving at each operating point; Formula (14) restricts each harvester starting from the agricultural machinery point and finally returning to the agricultural machinery point; Formula(15) indicates the delay time of the actual completion of the task at each farmland operation point; Formula (16) uses variables 0-1 to indicate whether the actual completion time of each farmland operation point is delayed; Formula (17) indicates the arrival time point of the harvester from point $h$ to point $j$; Formula (18) uses variables $0-1$ to indicate whether the time of the harvester arriving at point $j$ from point $h$ is earlier than the earliest time to start service allowed by point $j$; Formula (19) indicates that the task of the operation point completed by the distributed harvester according to the minimum demand within the time window, formula (20) indicates whether to assign model $i$ harvester to operation point $j$; Formula (21) represents the integer constraint. 


\section{MODEL SOLVING ALGORITHM DESIGN}

In 2000, the Elitist Non-dominated Sorting Genetic Algorithm was proposed to improve NSGA. NSGA-II has the advantages of low computational complexity, fast search speed, uniform distribution of solution set, and diversity of solutions without setting shared parameters (Chen et al., 2015). Therefore, NSGA-II is chosen to solve the trans-regional job scheduling model of combine.

\section{Chromosome Coding}

The chromosome coding structure in this paper is shown in Fig.1, each chromosome $S=\left(S^{1}, S^{2}, \cdots, S^{K}\right)$ corresponds to a scheduling scheme, each chromosome contains $K$ gene sites, and $K$ is the number of agricultural machinery points, $S^{k}$ represents a sequence of farmland operation points assigned by an agricultural machinery point $k, S^{k}=\left(S^{k 1}, S^{k 2}, \cdots, S^{k I}\right) I$ represents the number of types of harvesters; $S^{k i}=\left(S_{1}^{k i}, S_{2}^{k i}, \cdots, S_{l}^{k i}\right)$ represents the sequence of operation points assigned to the mode $i$ harvester at agricultural machinery point $k, S_{l}^{k i}$ represents the operating point sequence served by the $l$ th harvester (that is, the harvester $V_{l}^{k i}$ ) of the model $i$ at the agricultural machinery point $k$, $S_{l}^{k i}=\left(S_{1}^{k i}, S_{2}^{k i}, \cdots S_{N_{k}^{i}}^{k i}\right), N_{k}^{i}$ represents the number of model $i$ harvesters of the agricultural machinery point $k$.

\section{Initial Population Generation}

In order to ensure the feasibility and validity of the initial solution and accelerate the convergence speed of the algorithm, this paper adopts a construction algorithm based on heuristic priority rules to generate the initial population (Wang et al., 2015). The basic idea of the algorithm is as follows:

(1) Cluster of operation points. For the operation point $j$, if only one type of harvester can meet its operation requirements, then assign the model of harvester to it; if more than one type of machine is available for the operation point, then randomly assign a type that meets the requirements, so that each operation point is assigned a type that meets its harvesting requirements. According to the assignment models of the work point to cluster, so that the work points assigned the same type are classified into one class.

(2) Stratified sorting of operating points. For each type of operation point, if the time windows of all the operation points do not overlap, this type of operation point is a serial task, as long as the harvesting service is performed for each point in accordance with the time window order. If some or all of the time windows of each operation point overlap, there are parallel tasks. Due to the limited resources of the harvester, it may not be able to serve all parallel tasks at the same time, so there is a sequence of services for parallel operation points.

For each type of operation point, first sort it in ascending order from morning to night according to its required time window, let the number of layers of the top-ranked operation point is 1 , and take out each operation point $j$ in order, and determine whether $j$ and the next operation point are parallel tasks. If so, the two operation points are recorded as the same level. Otherwise the level is added by 1. Judge in turn until all operation points are layered. The lower the operating level, the higher the priority of harvesting service .

(3) Design of scheduling rules. Deploy the closest harvester to the operation point in priority. Assign the number of harvesters for each operation point according to the minimum demand. If there are no parallel tasks, then use rules 1 and 2 to assign harvesters to each operation point in chronological order; If there are parallel tasks, then use rules 1 and 2 to assign harvesters to operation points on each feasible path. 


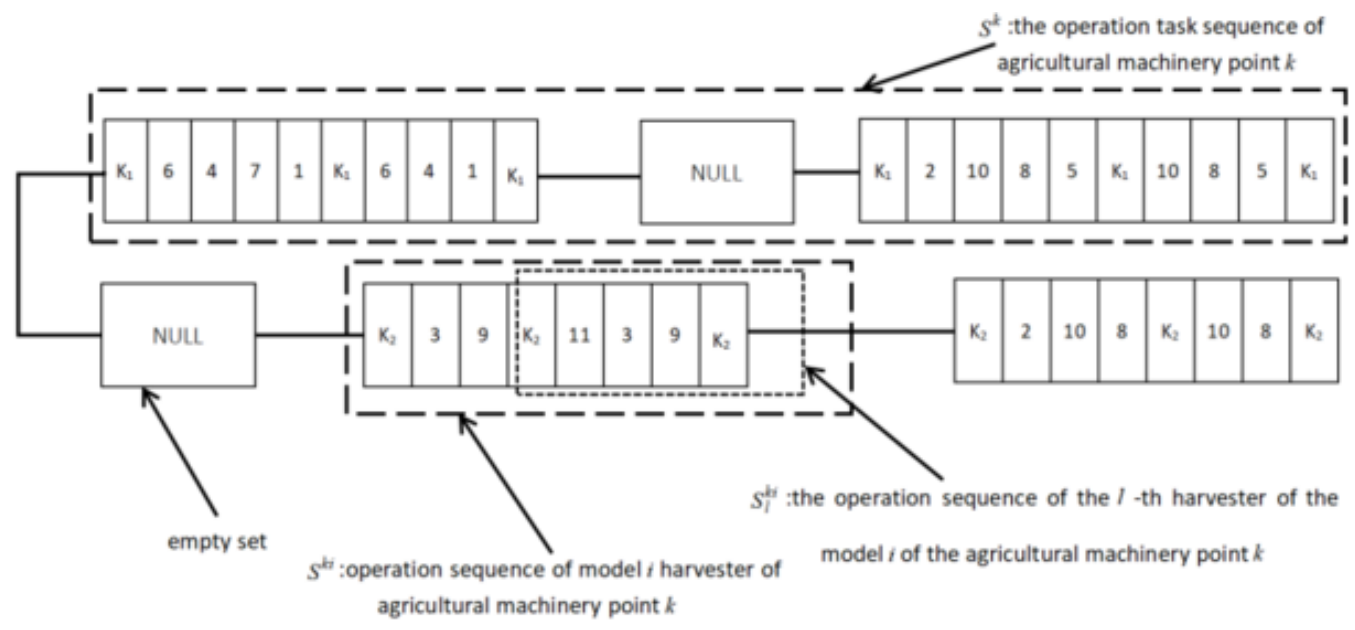

(4) Assignment of harvester based on combination rules. For each type of operation point, in its feasible path, first use rules 1 and 2 to assign the harvester for the first level operating point, if the harvester can be assigned for all operation points on the first level at the same time, record the allocation scheme. If the assignment cannot be completed, all feasible schemes of the operation points on the first level are recorded; then start from each scheduling scheme, search all feasible schemes of the operating points on the second level, and complete the search of the operating points of all levels in turn.

\section{Select Operator}

First of all, non-dominant ranking is performed according to multiple sub-objectives in the objective function of the population individuals; secondly, further evaluation is performed by calculating the crowding distance for individuals with the same non-dominant level; finally, integrate the evaluation value of Pareto's advantages and disadvantages and the evaluation value of crowding degree, and use binary tournament method to select and operate.

\section{Crossover Operator}

Due to the particularity of the chromosome coding method, this paper uses the sequence crossover method, that is, swapping the sequence of operating points accessed by agricultural machinery points in two parental individuals (Xu et al., 2013), as shown in Fig. 2.

Assuming two chromosome paternal individuals $P_{1}=\left(S_{1}^{1}, S_{1}^{2}, \ldots, S_{1}^{k}\right)$ and $P_{2}=\left(S_{2}^{1}, S_{2}^{2}, \ldots, S_{2}^{k}\right)$, randomly select a gene site for crossover, that is, to exchange sequences $S_{1}^{k}$ and $S_{2}^{k}$ of individuals $P_{1}$ and $P_{2}$, after crossing, two sub individuals $C_{1}$ and $C_{2}$ can be obtained.

Due to the encoding method of chromosomes, the agricultural operating points in the subindividuals may repeatedly appear in multiple model sequences after the exchange sequence, but only one type of machine can be assigned to each agricultural operating point, so it is necessary to delete the repeated operating points. After deletion, it will be found that the sub-individuals have lacked part of the operation points, so it is necessary to insert the missing operation points into the sequence of the chromosome. The specific insertion steps are as follows: 
Step 1: Determine the sequence of harvesters inserted at the operating point. First, the working time of each model $T_{i}, i \in N_{j}, T_{i}=\sum_{k=1}^{K} \sum_{l=1}^{N_{k}^{i}} T S_{l}^{k i}, k \in V^{k}$ is calculated for the set $N_{j}$ which meets the requirements of operation point $j, T S_{l}^{k i}$ represents the current total working time of harvester $V_{l}^{k i}$, which is the sum of the working hours of harvester sequence $V_{l}^{k i}$ at each operating point. Then sort $T_{i}$ in order from small to large, and select the model with short working time in priority. After determining the model $i$, sort the working time $T S_{l}^{k i}$ of each harvester of this model from small to large, and select the harvester with the shortest working time in priority. The minimum demand for this model is $R_{i j}$ for the operation point, so inserting operation point $j$ into $R_{i j}$ harvester sequences in turn.

Step 2: Determine the specific position where the operation point is inserted into a sequence. Supposing operation point $j$ is inserted between two adjacent operation points $(h, m)$ in a harvester's operation sequence $S_{l}^{k i}=(k i, 1,2, \cdots, k i)$ so obtain the harvester sequence, let $X(h, j, m)$ represents the total cost after insertion, $X(h, j, m)=\omega_{1} F_{1}+\omega_{2} F_{2}+\omega_{3} F_{3} . F_{1}$ is the total travel distance of the harvester after insertion in it, $F_{2}$ is the total waiting time of the harvester, $F_{3}$ is the total delay time of the operation task in the sequence, $\omega_{1}, \omega_{2}, \omega_{3}$ is the weighting coefficient, and the best position of operation point $j$ inserted into the sequence is the minimum position of $\min X(h, j, m)$

\section{Mutation Operator}

The mutation operation in the evolution process is to search the unknown space further and ensure the feasibility of the mutated chromosome at the same time. This paper uses a multi-mode mutation operator including sub-sequence segmentation, sub-sequence merging and sub-sequence partial exchange (Zhang et al., 2010). Randomly select a nonempty sequence $S^{k i}$ from chromosome $S=\left(S^{1}, S^{2}, \cdots, S^{K}\right)$ as the mutation gene position, and choose one of the three methods to perform the mutation operation. The basic process is shown in Fig. 3.

(1) Sub-sequence merging refers to selecting sub-sequence $S_{l}^{k i}$ and $S_{r}^{k i}$ of the two harvesters with the least amount of work from sequence $S^{k i}$ (Guo,2013). Assuming that the amount of work assigned to harvester $V_{l}^{k i}$ is less than that assigned to harvester $V_{r}^{k i}$, then the agriculture task points which are different from $S_{r}^{k i}$ in sub-sequence $S_{l}^{k i}$ are inserted into sub-sequence $S_{r}^{k i}$ in turn, and until $S_{l}^{k i}$ violates the constraint and no more any operation point is inserted, and the remaining work tasks are retained in sub-sequence $S_{l}^{k i}$ at the same time, If all the operation points of harvester $V_{l}^{k i}$ are merged to sub-sequence $S_{r}^{k i}$, then $S_{l}^{k i}$ is deleted which means that harvester $V_{l}^{k i}$ is not used.

(2) Sub-sequence segmentation refers to randomly selecting a sequence $S_{l}^{k i}$ from the sequence $S^{k i}$, and then inserting a $k i$ at a random position in the sub-sequence, that is, adding a harvester to complete the task, the sub-sequence is divided into two sub-sequences(Liu et al., 2012); if the segmented sub-sequence violates the maximum number constraint of harvesters, then select the harvester sequence with the minimum amount of work of this model and delete it from the chromosome

(3) Sub-sequence exchange refers to randomly selecting the operating points inside the two harvesters' sequences $S_{l}^{k i}$ and $S_{r}^{k i}$ for exchange, if the exchanged chromosome violates any of the constraints, the mutation is invalid, the original individual is retained without exchange. 
Figure 2. Crossover of chromosomal gene

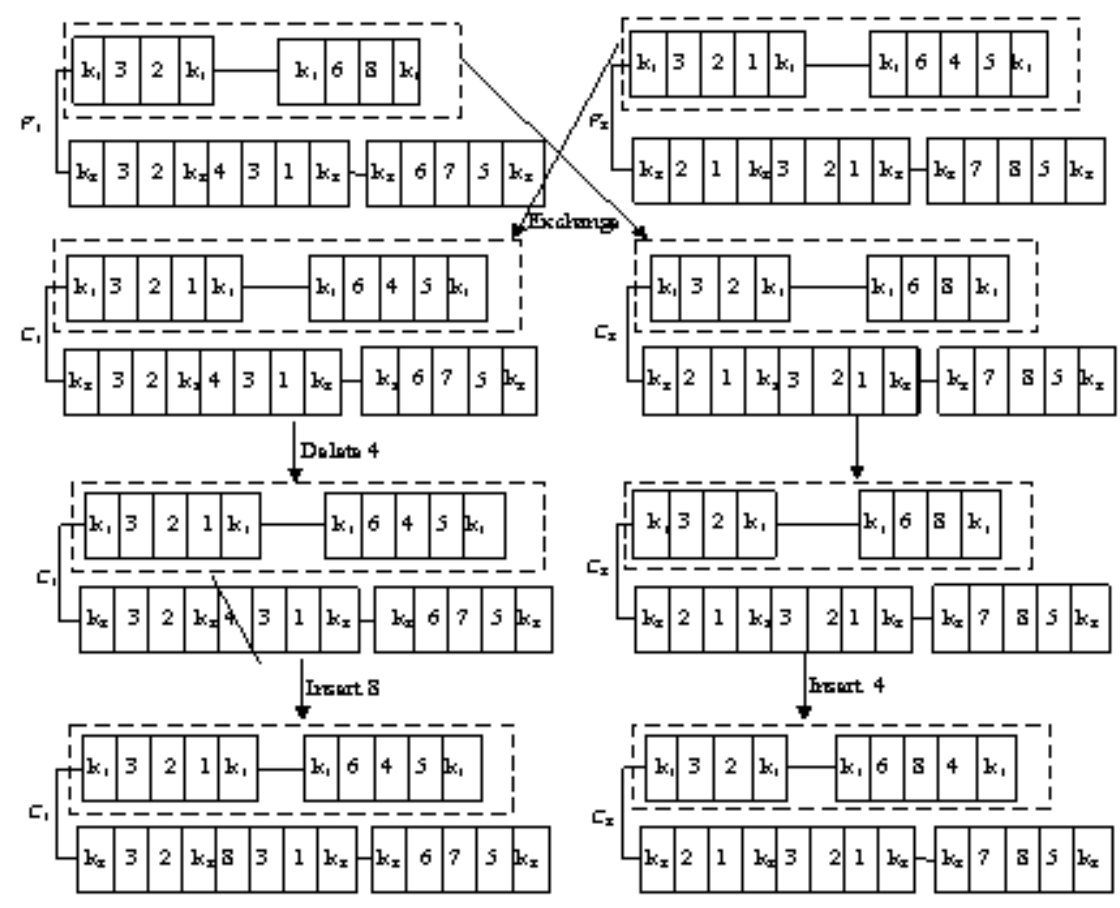

\section{ALGORITHM EVALUATION}

The paper combined with the actual situation of four agricultural machinery cooperatives in Wuchang City in 2019 to verify the model. The data came from a consultation with the Agricultural Machinery Bureau and an actual investigation. These agricultural machinery cooperatives had three types of combines: Kubota 4LZ-2.5 (M1), Kubota 4LBZ-172B (M2), and Lovol God GN70 (M3). There were 28 sets of three models in total. The planting area of rice was 39,655 Mu. The basic information of 50 operation sites was collected in the actual investigation operating point. The performance of each model of harvester was shown in Table 2, the locations of the cooperatives and the harvesters owned by cooperatives were shown in Table 3 .

Combined with the actual investigation, the harvester working time was set to $12 \mathrm{~h} /$ day, the transportation speed during the transfer process was $35 \mathrm{~km} / \mathrm{h}$, the transfer cost per unit distance was 2 yuan $/ \mathrm{km}$, and the waiting cost per unit time was $35 \mathrm{yuan} / \mathrm{h}$.

The paper used MATLAB language for algorithm programming. Setting the initial population size as 50 , the evolution algebra as 200 , the crossover probability as 0.8 , the mutation probability as 0.1 , and running the program on the computer with $2 \mathrm{~GB}$ of memory and Windows 10 system by MATLAB for many times, and finally obtained the Pareto set, as shown in Table 4.

After running the program many times, a set of Pareto solutions was selected, and the result of solving the multi-objective problem was the optimal solution set. According to the Pareto set, the optimal values of multiple objectives were obtained. Based on the practical survey data, the solution set gave the minimum deployment cost, the minimum number of harvesters, the shortest delay time and the maximum number of tasks completed on time. There were differences in index characteristics between different solutions, and decision makers can choose a solution in the solution set according to their actual needs. 
Figure 3. Schematic diagram of the composition of multi-mode mutation

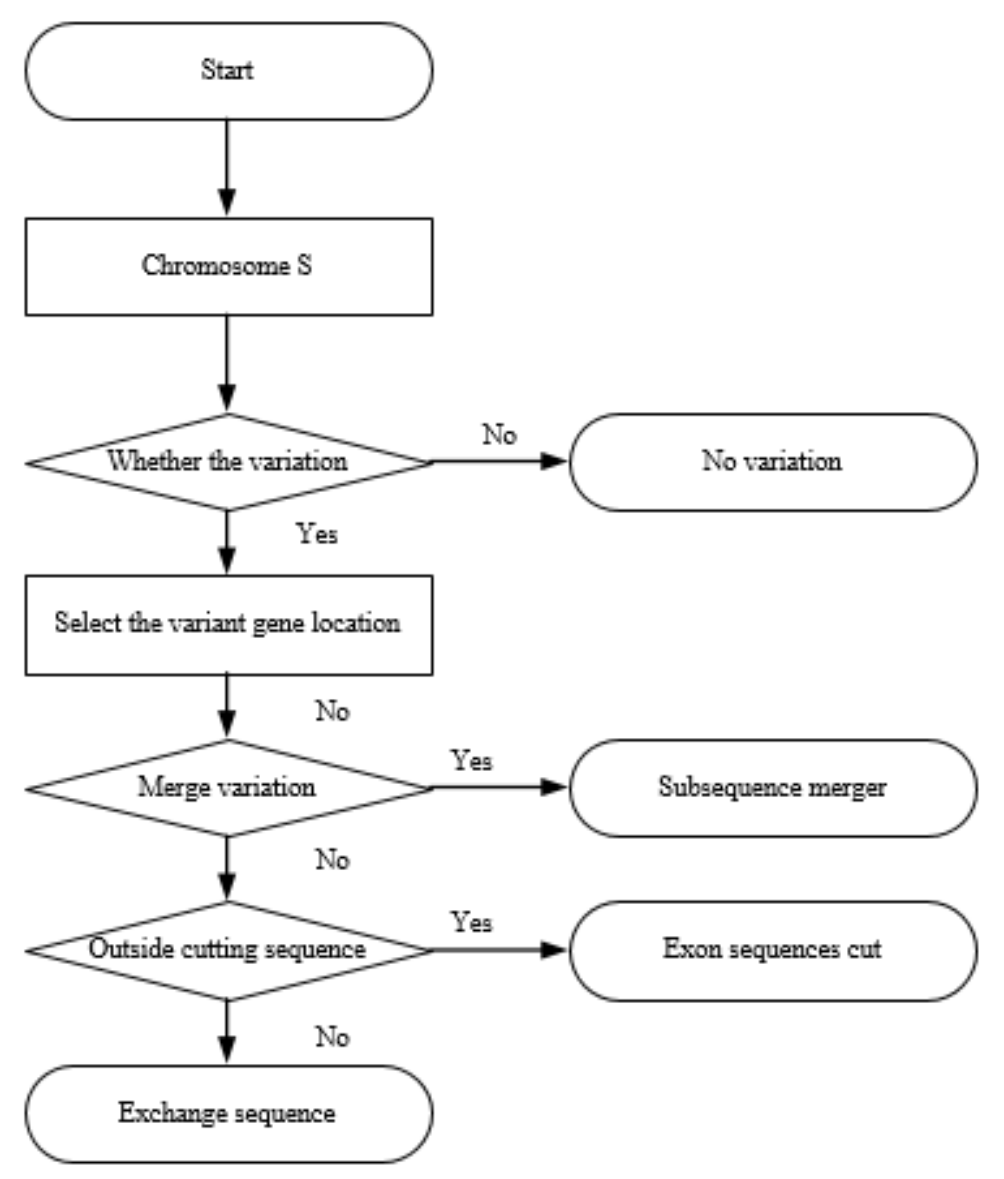

Through on-site investigation and communication of the four cooperatives, it was found that what the four cooperatives currently need most was to use fewer harvesters to complete their operations. Therefore, the paper chose the solution set of No. 10 (from table 4) to analyze, in the solution set, the minimum number of harvesters was 24 . The operation routes of the harvesters at four agricultural machinery points were shown in Fig. 4, Fig. 5, Fig. 6 and Fig. 7 respectively.

According to Table 5, on the premise of using the least number of harvesters, the total deployment distance was $6248.58 \mathrm{~km}$, and the total waiting time was $45 \mathrm{~h}$. All harvesters of agricultural machinery points K1 and K2 were used. One M2 harvester was not used in agricultural machinery point K3, and three M1 harvesters were not used in agricultural machinery point K4. The harvesting area of four agricultural machinery points were 12846.9 Mu, 13716.45 Mu, 9154.75 Mu and 3939 Mu respectively.

At the current stage, the four cooperatives did not have separate and specific records of delay time and deployment costs, so the number and area of combines were used to explain. The four Agricultural Machinery Cooperatives in Wuchang City had 28 harvesters with a harvesting area of $39655 \mathrm{Mu}$. The results showed that the harvesting area of 24 harvesters can reach $39657.1 \mathrm{Mu}$ after reasonable allocation. Compared with the practice, the results of the algorithm showed that the cooperative can use fewer harvesters to complete a larger area of harvesting tasks. 
Table 2. Performance of different types of combines

\begin{tabular}{|c|c|c|c|c|c|}
\hline $\begin{array}{c}\text { Model } \\
\text { number }\end{array}$ & $\begin{array}{c}\text { Feeding } \\
\text { mode }\end{array}$ & Power(Hp) & $\begin{array}{c}\text { Swath } \\
(\mathbf{m})\end{array}$ & $\begin{array}{c}\text { Cost } \\
(\text { yuan/unit })\end{array}$ & $\begin{array}{c}\text { Productivity } \\
(\mathbf{M u} / \mathbf{h})\end{array}$ \\
\hline M1 & Full feed & 68 & 2 & 520 & $3 \sim 7.95$ \\
\hline M2 & Half feed & 88 & 1.72 & 550 & $4.05 \sim 7.95$ \\
\hline M3 & Full feed & 170 & 4.57 & 580 & $9 \sim 18$ \\
\hline
\end{tabular}

Table 3. The location, combine type and quantity of each agricultural machinery point

\begin{tabular}{|c|c|c|c|c|c|}
\hline $\begin{array}{c}\text { Agricultural machinery } \\
\text { point number }\end{array}$ & Latitude $\left(^{\circ}\right)$ & $\begin{array}{c}\text { Longitude } \\
\left.{ }^{\circ}\right)\end{array}$ & M1 & M2 & M3 \\
\hline K1 & 45.084 & 127.084 & 3 & 1 & 3 \\
\hline K2 & 44.825 & 127.177 & 3 & 2 & 3 \\
\hline K3 & 45.024 & 127.443 & 1 & 4 & 2 \\
\hline K4 & 44.917 & 127.559 & 3 & 3 & 0 \\
\hline Total & & & 10 & 10 & 8 \\
\hline
\end{tabular}

According to the simulation results, using the scheduling model and the algorithm can solve the problem of trans-regional job-scheduling problem. The research results are conducive to optimizing the daily management of the cooperatives: according to the comprehensive research results, the cooperatives can decide whether to buy and when to buy a harvester. If the capacity of agricultural machinery is excessive, the cooperatives can consider whether to participate in socialized agricultural machinery services such as agricultural production trusteeship, which can not only ensure its own agricultural production, but also increase additional agricultural income, so as to maximize the use of harvester resources. Effectively reduce the total cost of dispatch, the cooperative can spend less expenditure to obtain higher production efficiency, reduce the ineffective consumption of harvesters and energy expenditures such as diesel; ensure the timely realization of crop harvest.

The feasibility and effectiveness of the model and algorithm were verified by empirical analysis. Trans-regional operation scheduling is a complex system engineering, which needs further improvement and perfection, mainly summarized as follows:

(1) According to the deployment model established in this study, the software of intelligent scheduling system for trans-regional operation could be developed in combination with $3 \mathrm{~S}$ technology and wireless communication technology, so as to realize the intelligent monitoring and scheduling of agricultural machinery for trans-regional operation.

(2) Agricultural production operations often contain multiple operational links and require a variety of agricultural machinery to complete the whole process of mechanized production. Therefore, the results of the paper could be extended to the formulation of trans-regional operation scheduling strategies for other agricultural machines in order to further improve the overall mechanization of agricultural production and reduce the total operating cost.

(3) When multiple objectives were considered in the selection of alternatives, decision makers can give different weights to the objectives according to the actual needs, such as analytic hierarchy process (AHP) and other evaluation methods to select the optimal solution from the Pareto solution. 
Table 4. The Pareto set

\begin{tabular}{|c|c|c|c|c|}
\hline Number & Deployment costs (yuan) & $\begin{array}{c}\text { Number of tasks } \\
\text { completed on time } \\
\text { (amount) }\end{array}$ & $\begin{array}{c}\text { Delay time at the } \\
\text { end of the operation } \\
\text { (h) }\end{array}$ & $\begin{array}{c}\text { Number of } \\
\text { harvester usage } \\
\text { (unit) }\end{array}$ \\
\hline 1 & 28302 & 45 & 34 & 26 \\
\hline 2 & 28543 & 47 & 30 & 28 \\
\hline 3 & 28026 & 47 & 25 & 27 \\
\hline 4 & 27937 & 44 & 36 & 26 \\
\hline 5 & 28435 & 47 & 20 & 28 \\
\hline 6 & 28365 & 46 & 27 & 25 \\
\hline 7 & 27539 & 42 & 28 & 26 \\
\hline 8 & 29201 & 46 & 32 & 24 \\
\hline 9 & 29136 & 47 & 30 & 26 \\
\hline 10 & 27956 & 43 & & 26 \\
\hline
\end{tabular}

Figure 4. The route map of combines of agricultural machinery point K1

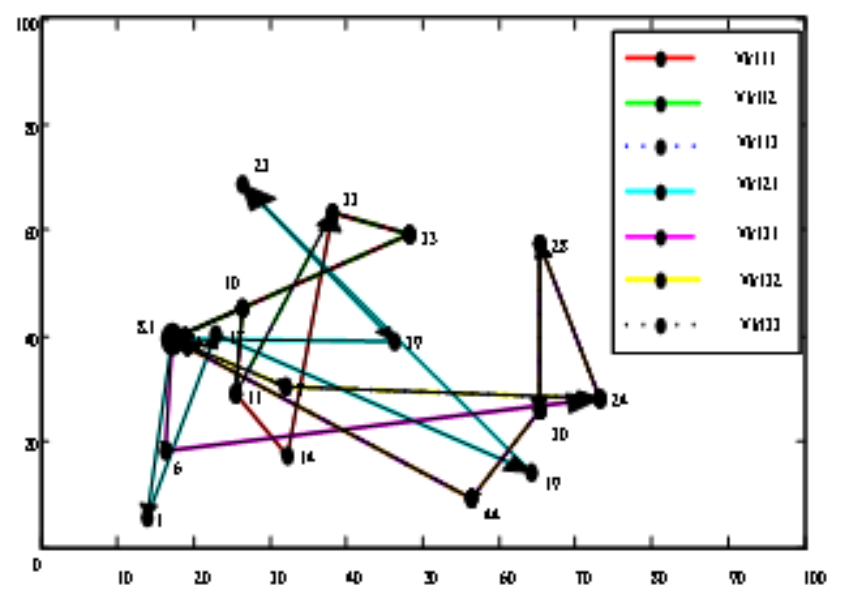

\section{CONCLUSION}

Based on the idea of agricultural machinery resources integration, combined with the characteristics and requirements of trans-regional operation scheduling of combine of agricultural machinery cooperatives, this paper analyzed the current trans-regional operation mode of combine harvester, established the trans-regional operation scheduling model of combine based on the cooperation and sharing of multiple agricultural machinery cooperatives, designed the model solving algorithm, and analyzed empirically with the situation of the rice harvest in Wuchang city of Heilongjiang Province in China. The rationality and effectiveness of the model and algorithm were verified by empirical analysis. The main conclusions were as follows:

(1) The characteristics of trans-regional operation scheduling of combine were analyzed deeply, and was abstracted as a multi depot, multi vehicle type and time window constrained harvester 
Figure 5. The route map of combines of agricultural machinery point K2

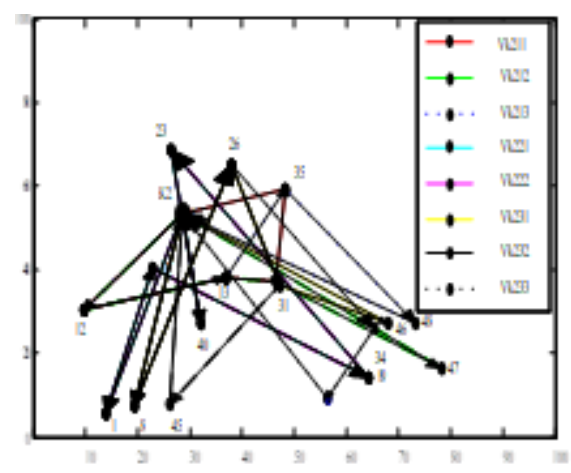

Figure 6. The route map of combines of agricultural machinery point K3

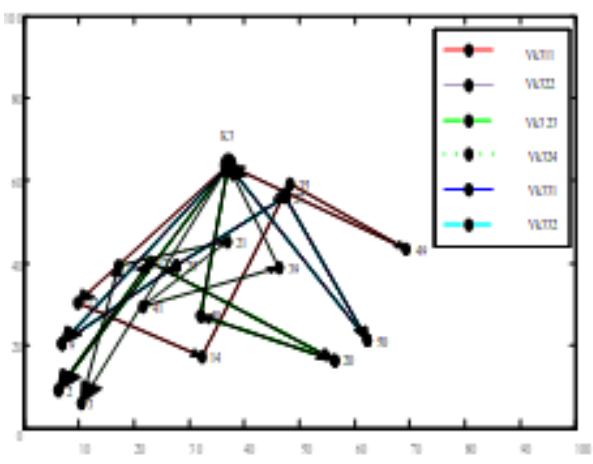

Figure 7. The route map of combines of agricultural machinery point K4

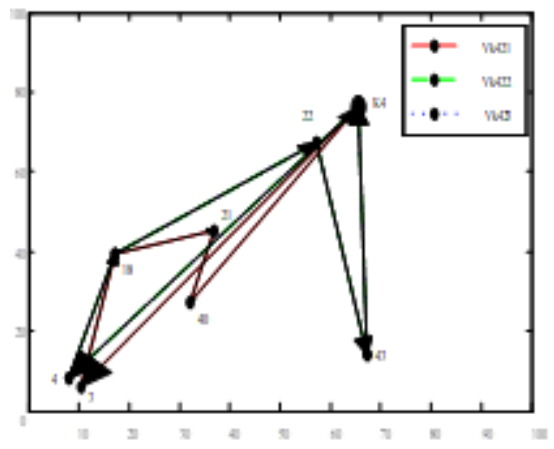

resource allocation problem. Combined with path optimization and job shop scheduling theory, each job point was regarded as a workpiece to be processed, and the harvester was regarded as a mobile processing station. The start time and end time of each operation point were analyzed with the idea of punctuality, from the perspective of farmers and owners, a low-cost, just in time scheduling model for trans-regional operation of combine was established.

(2) Based on the analysis of multiple scheduling algorithms, NSGA-II, a non dominated sorting genetic algorithm with elite retention strategy, was selected to design the model solving algorithm. 
Table 5. A pareto optimal scheduling scheme

\begin{tabular}{|c|c|c|c|c|}
\hline $\begin{array}{c}\text { Harvester serial } \\
\text { number }\end{array}$ & Working route & $\begin{array}{c}\text { Harvest } \\
\text { Area } \\
\text { (Mu) } \\
\end{array}$ & $\underset{(\mathrm{km})}{\operatorname{Deployment} \text { distance }}$ & $\underset{\text { (h) }}{\text { Waiting time }}$ \\
\hline$V_{1}^{11}$ & K1-10-11-14-33-35-K1 & 745.3 & 191.64 & 0 \\
\hline$V_{2}^{11}$ & K1-10-11-33-35-K1 & 764.3 & 254.67 & 3 \\
\hline$V_{3}^{11}$ & K1-10-11-33-35-K1 & 764.4 & 254.67 & 0 \\
\hline$V_{1}^{12}$ & K1-1-17-19-23-39-K1 & 756.9 & 328.40 & 4 \\
\hline$V_{1}^{13}$ & K1-6-24-28-30-44-K1 & 2615 & 356.27 & 0 \\
\hline$V_{2}^{13}$ & $\mathrm{~K} 1-7-24-28-30-44-\mathrm{K} 1$ & 2350.5 & 395.82 & 4 \\
\hline$V_{3}^{13}$ & K1-7-24-28-30-44-K1 & 2350.5 & 395.82 & 0 \\
\hline$V_{1}^{21}$ & $\mathrm{~K} 2-12-13-34-35-\mathrm{K} 2$ & 721.75 & 189.34 & 5 \\
\hline$V_{2}^{21}$ & $\mathrm{~K} 2-12-13-34-47-\mathrm{K} 2$ & 721.75 & 278.29 & 0 \\
\hline$V_{3}^{21}$ & $\mathrm{~K} 2-12-13-35-48-\mathrm{K} 2$ & 744.75 & 157.32 & 0 \\
\hline$V_{1}^{22}$ & K2-1-17-19-23-40-K2 & 886.9 & 268.35 & 5 \\
\hline$V_{2}^{22}$ & K2-1-17-19-23-40-K2 & 887.2 & 268.35 & 4 \\
\hline$V_{1}^{23}$ & K2-8-26-31-46-K2 & 2204 & 332.18 & 0 \\
\hline$V_{2}^{23}$ & $\mathrm{~K} 2-8-26-31-45-\mathrm{K} 2$ & 2243 & 364.18 & 0 \\
\hline$V_{3}^{23}$ & $\mathrm{~K} 2-8-26-30-44-\mathrm{K} 2$ & 2350 & 273.47 & 0 \\
\hline$V_{1}^{31}$ & K3-12-14-35-49-K3 & 746.75 & 259.46 & 8 \\
\hline$V_{2}^{32}$ & K3-2-17-20-40-K3 & 979 & 421.67 & 0 \\
\hline$V_{3}^{32}$ & K3-2-17-20-40-K3 & 979 & 421.67 & 0 \\
\hline$V_{4}^{32}$ & K3-3-18-21-41-39-K3 & 954 & 124.28 & 4 \\
\hline$V_{1}^{33}$ & K3-9-25-27-50-K3 & 2098 & 143.14 & 0 \\
\hline$V_{2}^{33}$ & K3-9-25-27-50-K3 & 2098 & 143.14 & 0 \\
\hline$V_{1}^{42}$ & K4-3-18-21-40-K4 & 977 & 102.3 & 5 \\
\hline$V_{2}^{42}$ & K4-4-18-22-43-K4 & 765 & 324.15 & 0 \\
\hline$V_{3}^{42}$ & K4-4-18-22-43-K4 & 765 & 324.15 & 3 \\
\hline
\end{tabular}


Combining with the characteristics of this model, the chromosome coding method, the generation of initial population, crossover and mutation process of the algorithm were designed and improved in detail.

(3) Combined with four agricultural machinery cooperatives in Wuchang City, Heilongjiang Province, China, the simulation analysis was carried out and the operation scheduling of four agricultural machinery cooperatives was given. The experimental results showed that compared with the current scheme, the optimized scheme used the least number of harvesters to complete the task, improved the efficiency of harvester and realized the maximum utilization of resources. It could reduce the total production cost and save the operation time. At the same time, a reasonable operation route was planned for each harvester dispatched, and appropriate operation tasks were allocated to ensure the balance of harvester workload.

The innovation of this paper: the multi-objective programming model of trans-regional operation scheduling for combine was established by applying path optimization and job shop scheduling theory. The model was solved based on NSGA-II algorithm, and the chromosome coding, initial population generation, crossover and mutation process of the algorithm were designed in detail.

\section{ACKNOWLEDGMENT}

This work were supported by the Open Project of Key Laboratory (Modern Agricultural Equipment Technology in cold region of North China) (Grant No.KF18-02), Heilongjiang Province "Ten Thousand Million" Project(Grant No.2019ZX14A04). 


\section{REFERENCES}

Basnet, C. B., Foulds, L. R., \& Wilson, J. M. (2006). Scheduling contractors' farm-to-farm crop harvesting operations. International Transactions in Operational Research, 13(1), 1-15. doi:10.1111/j.14753995.2006.00530.x

Bochtis, Dionysis \& Vougioukas, Stavros \& Ampatzidis, Yiannis \& Tsatsarelis, Constantinos. (2007). Field Operation Planning for Agricultural Vehicles: A Hierarchical Modeling Framework. Agricultural Engineering International: the CIGR Journal of Scientific Research and Development. IX.

Chen, H., Jiang, Z., Zuo, L., \& Zhang, Y. (2015). Multi-objective flexible job-shop scheduling problem based on NSGA-II with close relative variations. Transactions of the Chinese Society for Agricultural Machinery, $46(4), 344-350$.

D’Urso, G., Smith, S. L., Mettu, R., Oksanen, T., \& Fitch, R. (2018). Multi-vehicle refill scheduling with queueing. Computers and Electronics in Agriculture, 144, 44-57. doi:10.1016/j.compag.2017.11.014

Ferrer, J. C., Cawley, A. M., Maturana, S., Toloza, S., \& Vera, J. (2008). An optimization approach for scheduling wine grape harvest operations. International Journal of Production Economics, 112(2), 985-999. doi:10.1016/j. ijpe.2007.05.020

Foulds, L. R., \& Wilson, J. M. (2005). Scheduling operations for the harvesting of renewable resources. Journal of Food Engineering, 70(3), 281-292. doi:10.1016/j.jfoodeng.2003.12.009

Guan, S., Nakamura, M., Shikanai, T., \& Okazaki, T. (2009). Resource assignment and scheduling based on a two-phase metaheuristic for cropping system. Computers and Electronics in Agriculture, 66(2), 181-190. doi:10.1016/j.compag.2009.01.011

Guo, J. (2013). Research on dynamic vehicle routing optimization method of cigarette logistics distribution with time window (Doctoral thesis). Beijing Jiaotong University. Retrieved from http://h-s.kns.cnki.net.neau.vpn358. $\mathrm{com} / \mathrm{kcms} /$ detail/detail.aspx?FileName=101411063.nh\&DbName=CMFD2014

Li, H., Yao, G., \& Chen, L. (2008). Farm Machinery Monitoring and Scheduling System Based on GPS, GPRS and GIS. Nongye Gongcheng Xuebao (Beijing), 24, 119-122.

Liao, X., Gao, Y., Jiang, B., \& Huang, G. (2008). Development situation and prospect about cross-region harvest task. Nong-ji-hua Yanjiu, (2), 10-14.

Liu, A.-J \& Yang, Yorke \& Cheng, W.-M \& Xing, Q.-S \& Lu, H. \& Zhao, X.-H \& Zhang, Y.-D \& Zeng, Qihang \& Yao, H.. (2012). Improved NSGA for complex manufacturing environment. Jisuanji Jicheng Zhizao Xitong/ Computer Integrated Manufacturing Systems, CIMS. 18. 2446-2458.

Pilla, V. L., Rosenberger, J. M., Chen, V., Engsuwan, N., \& Siddappa, S. (2012). A multivariate adaptive regression splines cutting plane approach for solving a two-stage stochastic programming fleet assignment model. European Journal of Operational Research, 216(1), 162-171. doi:10.1016/j.ejor.2011.07.008

Seyyedhasani, H., \& Dvorak, J. S. (2018). Reducing field work time using fleet routing optimization. Biosystems Engineering, 169, 1-10. doi:10.1016/j.biosystemseng.2018.01.006

Sheridan, P. K., Gluck, E., Guan, Q., Pickles, T., Balc1og $₹$ lu, B., \& Benhabib, B. (2013). The dynamic nearest neighbor policy for the multi-vehicle pick-up and delivery problem. Transportation Research Part A, Policy and Practice, 49, 178-194. doi:10.1016/j.tra.2013.01.032

Tan, Q., Wang, L., \& Zhong, D. (2016). Application of NSGA-II in multi-objective route optimization of underground mine's transportation. Gold Science and Technology, 24(2), 95-100.

Tian, J. (2007). Modeling and optimization methods for multi-UAV cooperative reconnaissance mission planning problem (Masters' thesis). National University of Defense Technology. Retrieved from http://h-s.kns.cnki.net. neau.vpn358.com $/ \mathrm{kcms} /$ detail/detail.aspx $?$ FileName=2008098729.nh $\&$ DbName $=$ CDFD2009

Wang, C., Ye, J., Zeng, C., Xia, Y., \& Luo, B. (2015). Multi-objective optimum design of high specific speed mixed-flow pump based on NSGA-II genetic algorithm. Transactions of the Chinese Society of Agricultural Engineering, 31(18), 100-106. 
Wang, T., Liu, F., Gao, Y., Hu, N., \& Zhang, Y. (2020). Precision scheduling of Beidou agricultural machinery based on genetic algorithm and WiFi clustering algorithm. Journal of Jiangsu University (Natural Science Edition), 41(4), 426-433 + 445 .

Wang, W. (2019).Research on model and algorithm of agricultural machinery scheduling problem with time window (Masters' thesis). Zhejiang University. Retrieved from http://h-s.kns.cnki.net.neau.vpn358.com/kcms/ detail/detail.aspx?FileName $=1019079037$. nh $\&$ DbName $=$ CMFD2020

Wang, X., Jia, Y., Cai, J., \& Jin, Z. (2020). Joint optimization of resource allocation and operation scheduling of Railway Container Central Station. Control and Decision Making, 1-10.

Wang, Y. (2013). Cotton-picker cross-regional scheduling algorithm research and implementation (Doctoral thesis). Shihezi University. Retrieved from http://kns.cnki.net/kns/detail /detail.aspx?FileName=1013366176. nh\&DbName $=$ CMFD2014

Wang, Z., Chen, L., \& Liu, Y. (2010). Design and implementation of agricultural machinery monitoring and scheduling system. Computer Engineering, 169(4), 544-550.

Wu, C. \& Cai, Y. \& Luo, M. \& Su, H. \& Ding, L.. (2013). Time-windows based temporal and spatial scheduling model for agricultural machinery resources. Nongye Jixie Xuebao/Transactions of the Chinese Society for Agricultural Machinery. 44. 237-241+231. . 10.6041/j.issn.1000-1298.2013.05.041

Xu, B., Chen, L., Xu, M., \& Tan, Y. (2013). Path planning algorithm for plant protection UAV in multiple operation areas. Transactions of the Chinese Society for Agricultural Machinery, 48(02), 75-80.

Yang, Y., Wu, Y., Yao, Y., Ren, Z., \& Gao, Y. (2013). Research and application of heuristic algorithm for vehicle routing problem with time window. Journal of Computer Applications, 33(S1), 59-61.

Zhang, C., Zhao, Y., Chen, F., \& Ma, X. (2010). Multi-objective optimum design of dry-type air-core reactor based on improved NSGA-II algorithm. Proceedings of the CSEE, 30(18), 115-121.

Zhang, F., Teng, G., Ma, J., \& Chang, S. (2012). Farm machinery scheduling and allocating based on heuristic priority rules. Transactions of the Chinese Society of Agricultural Engineering. 28(10), 78-85. doi:10.3901/ JME.2012.11.078

Zhang, X. (2006). Study on the Service System in Trans-regional Harvesting Wheat Using Combine Harvester (Doctoral thesis). China Agricultural University. Retrieved from https://d.wanfangdata.com.cn/details/detail. do? type $=$ degree $\& i d=Y 940168$.

Zhao, G., \& Liu, Y. (2019). A Study on the Dynamic Scheduling Algorithm in the Workshop of Agricultural Machinery Steering. Electrical and Mechanical Engineering, 36(09), 980-984.

Zhao, S. (2013). Research on multi-objective intelligent algorithm based on elite set selection and expansion strategy (Masters' thesis). South China University of Technology. Retrieved from https://kns.cnki.net/kcms/ detail/detail.aspx?FileName $=1014153449$. nh $\&$ DbName=CDFD2014 
Ma Li was born in Heilongjiang Province, China in 1976. She received Ph. D in Agricultural mechanization engineering from Northeast Agricultural University, Harbin, China in January 2010. She is working as associated professor in Engineering College in Northeast Agricultural University. Her current research interests include Agricultural System Engineering and Industrial Engineering.

Yidi Wang received her B.Admin degree in Business Administration from Beijing Technology and Business University, China, in 2019. Currently, she is a graduate student in Management Science and Engineering from Northeast Agricultural University, China. Her research interests include data analysis.

Meiqiong Ma received her M.Admin degree in Management Science and Engineering from Northeast Agricultural University, China, in 2017. Her research interests include algorithm research.

Bai Jiyun was born at Heilongjiang Province, China, in 1979. She received Ph.D in Control Science and Control Engineering from Harbin Institute of Technology, Harbin, China, in January 2014. She is working as associated professor in College of Science in Northeast Agricultural University. Her current research interests include machine learning, intelligent algorithm, and smart agriculture. 